\title{
Echocardiography for assessment of left atrial stasis and thrombosis - three cases report
}

\author{
Ionela Tanasa ${ }^{1}$, Raluca Arhirii ${ }^{1}$, Radu $\mathrm{Popa}^{2,3}$, Antoniu Petriş ${ }^{\star, 1,2}$ \\ ${ }^{1}$ Cardiology Clinic, "Sf. Spiridon" University County Emergency Hospital , laşi, Romania, "'Grigore T. \\ Popa" University of Medicine and Pharmacy Iași, Romania, ${ }^{3}$ Vascular Surgery Clinic, "Sf. Spiridon" \\ Universitay County Emergency Hospital, Iași, Romania
}

\begin{abstract}
We report three cases of chronic atrial fibrillation without anticoagulation admitted for acute lower limb ischemia in which embolic source was left atrial appendage (LAA) thrombus revealed only by transesophageal echocardiography (TEE). Transthoracic echography (TTE) revealed left atrial dilatation (LA) with no contrast or intracavitary mass. TEE revealed LA with spontaneous echographic contrast, low ostial emptying velocities and congenital morphology of LAA with older and organized thrombus or with echolucent centre (" beard-beark sign of recent and growing thrombus") and thoracic aorta atheroma plaques. They were discharged on acenocumarol/ novel oral anticoagulant therapy. Two-dimensional echocardiography should be recommended in all patients with periprocedural acute peripheral embolism and TEE only in selected cases. Very high incidence of acute embolic ischemia is probably due to lack of preoperative anticoagulation more than to nontherapeutical anticoagulation (international normalized ratio INR<2).
\end{abstract}

Keywords: atrial fibrillation, left atrial, thrombosis, acute limb ischemia

\section{Introduction}

Thromboembolism secondary to atrial fibrillation (AF) accounts for $25 \%$ of all strokes [1], but arterial embolism of the extremities is not as rare and especially not as benign as it was considered in the past and contemporary data regarding this problem are sparse. The cause of systemic embolism is left atrial stasis and thrombosis [2].

We report three cases of chronic AF without anticoagulation admitted for acute lower limb ischemia in which the embolic source was left an appendage (LAA) thrombus revealed only by transesophageal echocardiography (TEE).

Received: April 2015; Accepted after review: June 2015; Published: June 2015.

${ }^{*}$ Corresponding author: Antoniu Petriş, "Grigore T. Popa" University of Medicine and Pharmacy lași, 16 Universitatii str. Iasi, 700115, Romania .

E-mail: antoniu.petris@yahoo.ro

\section{Cases presentations}

First case

A 66 year-old man, hypertensive with chronic AF without anticoagulation, type 2 diabetic, ex-smoker, with prior stroke and a history of primary embolectomy of left superficial femoral artery four years ago free of postsurgery symptoms, without anticoagulation until actual admission with a new episode of acute ischemia in the same territory. Immediate embolectomy was successfully done under local anesthetic with uncomplicated evolution.

We routinely examine by echocardiography all patients to find the source of embolism. TTE revealed a mild dilatation of LA with no contrast or intracavitary mass and normal left ventricular shape and contractility and moderate mitral regurgitation. TEE revealed LA with light spontaneous echocardiographic contrast (SEC), low ostial emptying velocities $<40 \mathrm{~cm} / \mathrm{sec}$ (Figure 1) and unilobar LAA with thrombus $(2 \mathrm{~cm} / 1.5 \mathrm{~cm})$ 
(Figure 2) with echolucent center ("beard-beak sign of recent and growing thrombus") [3], protruding to LA during systole (Figure 3), few thin thoracic aorta plaques. He was discharged on acenocumarol therapy.

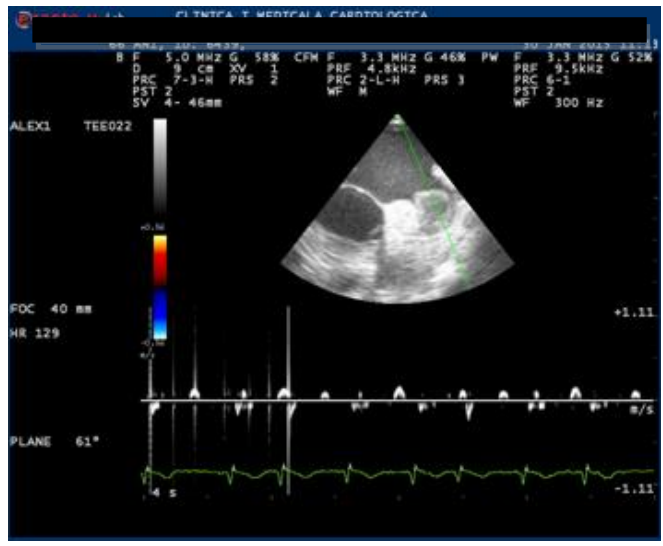

Fig. 1. Transesophageal echocardiography showing low ostial emptying velocities

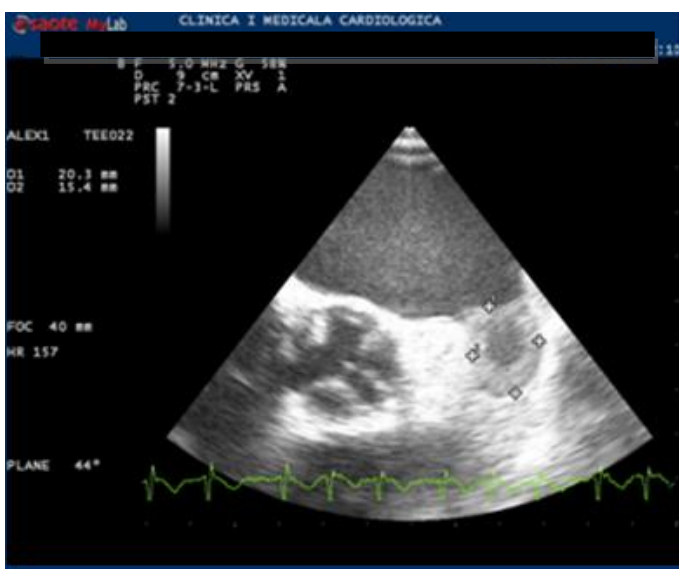

Fig. 2. Transesophageal echocardiography showing thrombus with echolucent center

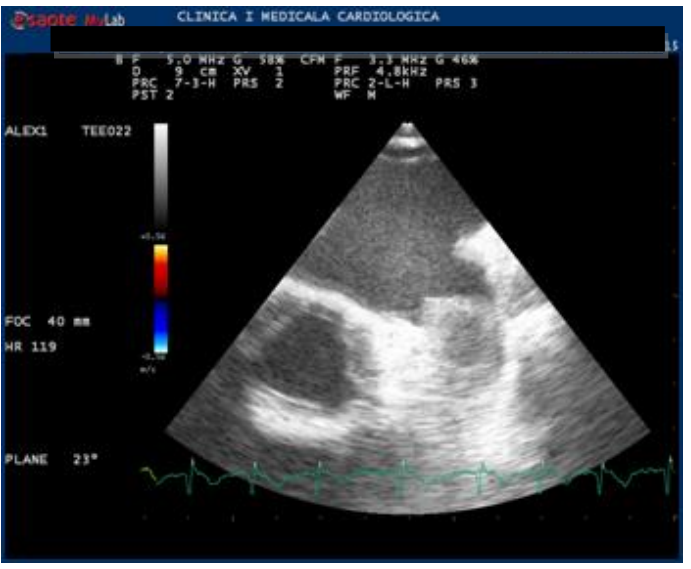

Fig. 3. Transesophageal echocardiography showing thrombus with echolucent center protruding to LA during systole

\section{Second case}

A 66 year-old woman, hypertensive with chronic AF without anticoagulation, with prior stroke, obese, alcoholic, presented with acute ischemia of the right common and superficial femoral artery.

Immediate embolectomy was successfully done under local anesthetic with uncomplicated evolution also.

TTE revealed a moderate LA dilation with no contrast (Figure 4) or intracavitary mass and left ventricular hypertrophy and normal contractility. TEE revealed LA with dense SEC (Figure 5), low ostial emptying velocities $<20 \mathrm{~cm} / \mathrm{sec}$ and LAA with 2 lobes and small thrombi in both lobes (Figure 6) and one filamentous thrombus $16 / 6 \mathrm{~mm}$ at the ostium of LAA, few thin thoracic aorta plaques but light SEC. She was discharged on dabigatran therapy.

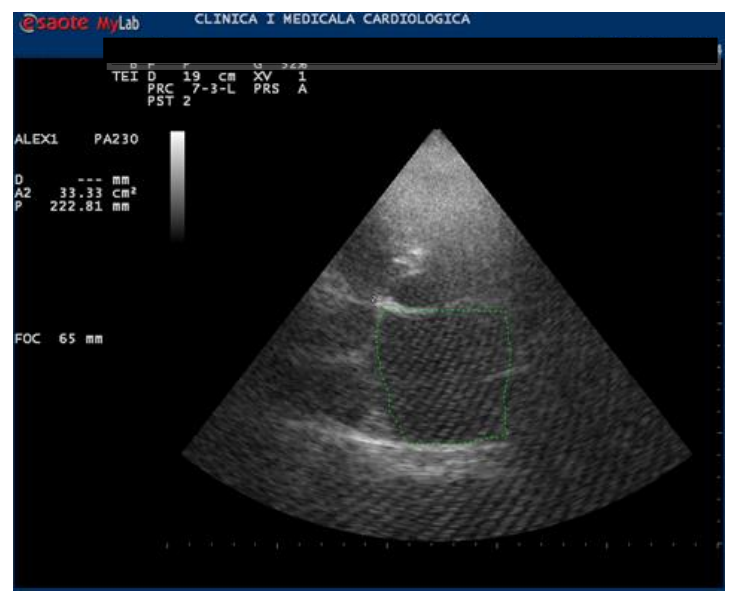

Fig. 4. Transthoracic echocardiography showing moderate LA dilatation with no contrast

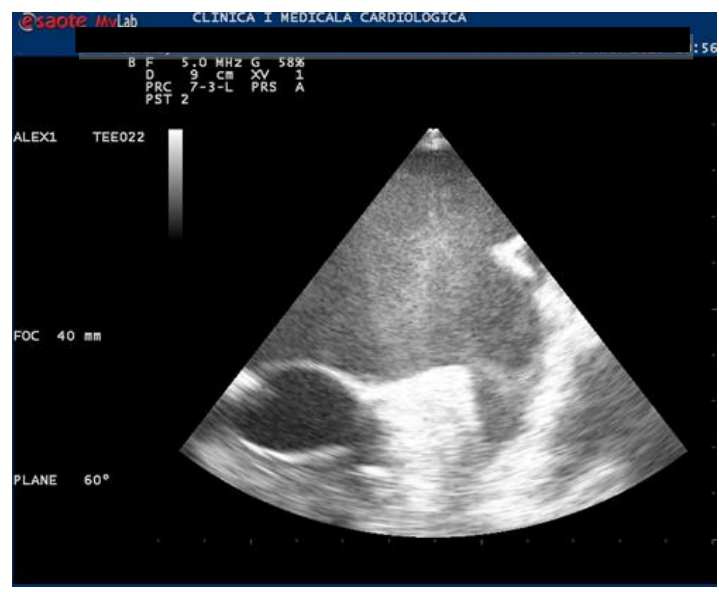

Fig. 5. Transesophageal echocardiography showing LA with dense SEC 


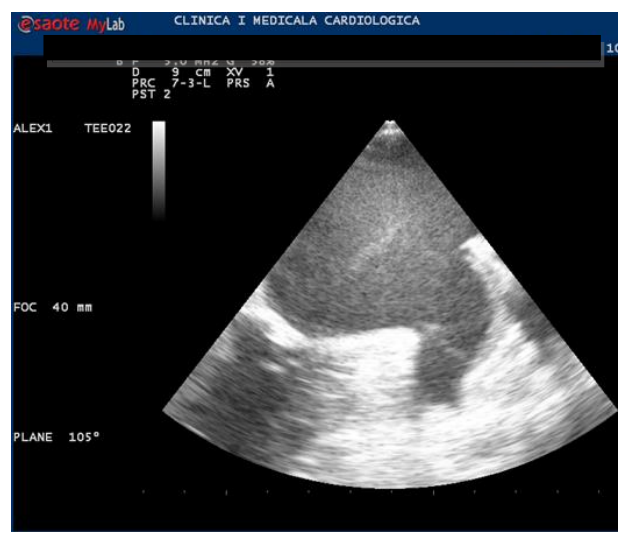

Fig. 6. Transesophageal echocardiography showing LA with dense SEC and small thrombi in bilobated LAA

\section{The third case}

A 67 year-old man, hypertensive with chronic AF without anticoagulation, with prior stroke, overweight, type 2 diabetic presented with acute ischemia of the right common and superficial femoral artery. Immediate embolectomy was successfully done under local anesthetic and postoperatory evolution was marked by an episode of silent inferolateral ischemia normalized with double oral antiaggregant therapy along with anticoagulation.

TTE revealed a moderate LA dilation with no contrast (Figure 7), but possible intracavitary mass and left ventricular hypertrophy with normal contractility. TEE revealed LA with dense SEC and thrombus 15 $\mathrm{mm}$ along (Figure 8) the posterior LA wall near interatrial septum (Figure 9), unilobar LAA (Figure 10) with low ostial emptying velocities $<20 \mathrm{~cm} / \mathrm{sec}$ and thrombus $8 \mathrm{~mm}$ and few nonembolic thin thoracic aorta plaques. He was discharged on acenocumarol therapy.

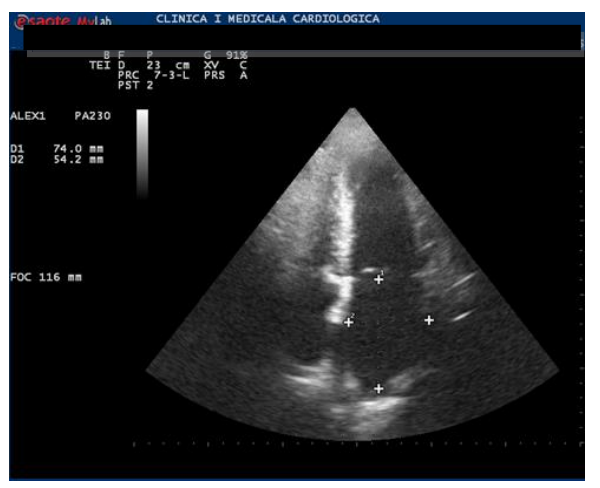

Fig. 7. Transthoracic echocardiography showing moderate LA dilatation with no contrast

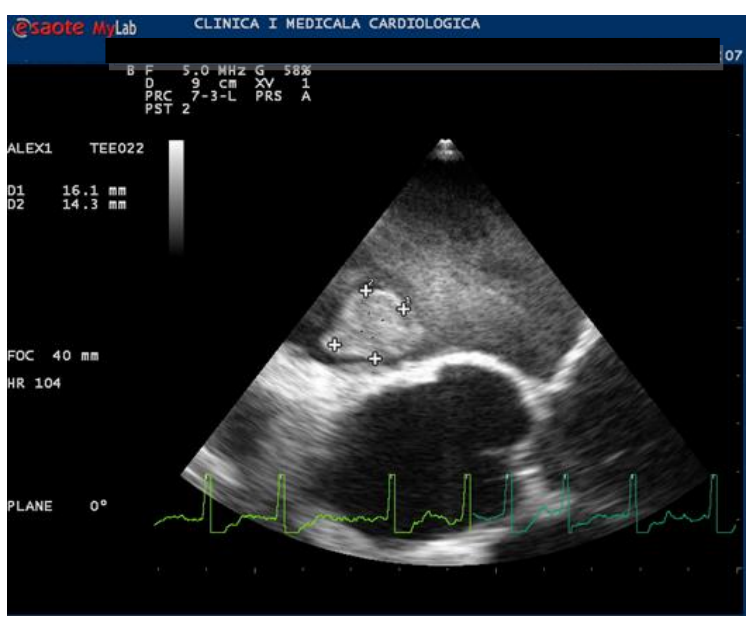

Fig. 8. Transesophageal echocardiography showing dense SEC and thrombus $15 \mathrm{~mm}$ along the posterior LA wall near interatrial septum

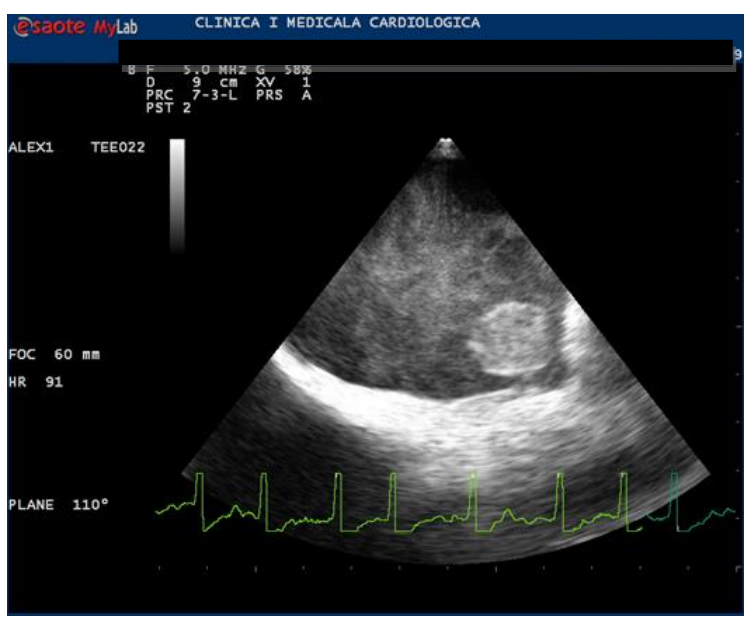

Fig. 9. Transesophageal echocardiography showing dense SEC and thrombus along the posterior LA wall near interatrial septum

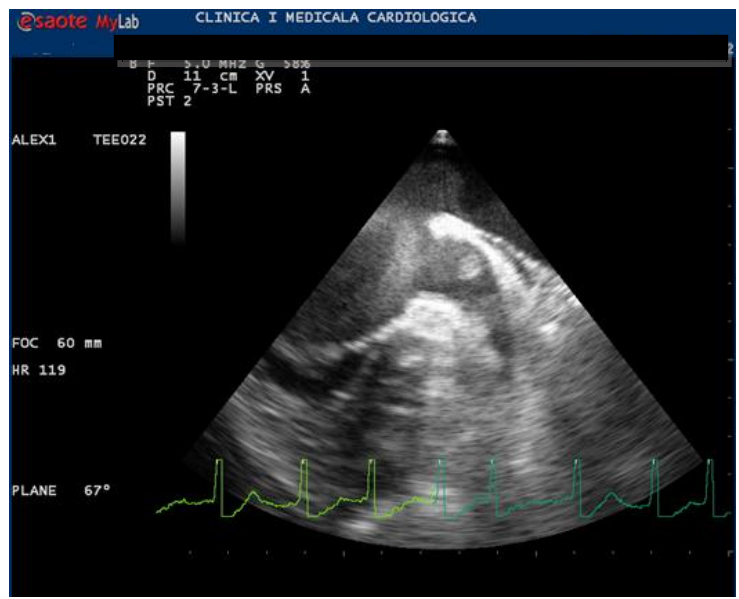

Fig. 10. Transesophageal echocardiography showing LA with dense SEC and small thrombi in unilobar LAA 


\section{Discussions}

$\mathrm{AF}$ is a frequent arrhythmia in the elderly population (age $>60$ years) and is the most common sustained cardiac arrhythmia after 70 years which means that is found in 1 to $5 \%$ of the general population between 60 to 69 years old, more frequent in men than in women [4].

AF is characterized by disorganized atrial depolarization without effective atrial contraction (irregular contraction) and irregularly irregular due to ventricular response concealed conduction AF is not life-threatening by itself but sometimes requires emergency treatment for hemodynamic alterations or can lead to systemic embolism.

AF has higher incidence in patients with LA and LAA dysfunction. The left atrial appendage (LAA) is the main location of thrombus formation well correlated with its morphology predominantly in patients with nonvalvular AF.

There are also rare cases of paradoxical thrombus from right atrium through patent foramen ovale (PFO) [5].

There are well established risk parameters for thrombus formation including CHADS(2) score (congestive heart failure, hypertension, age >75 years, diabetes, and previous stroke/transient ischemic attack) and CHA(2)DS(2)-VASc score (congestive heart failure, hypertension, age 75 years or older, diabetes mellitus, previous stroke or transient ischemic attack, vascular disease, age 65 to 74 years, female) $[1,6]$ and other independent risk predictors $[1,7,8]$ like complex LAA shape particularly more than 2 lobes, remodeling of LAA and LA [9, 10] (dilatation > $45 \mathrm{~cm}$, stretching, atrophy of pectinated muscles encased in endocardial fibroelastosis with chronic AF, emptying LAA velocities < $20 \mathrm{~cm} / \mathrm{sec}$, spontaneous echocardiographic contrast, thrombus) and in discussion inflammation markers like fibrinogen and reactive $\mathrm{C}$ protein.

The gold-standard modality for assessment of SEC and thrombus in the LAA is echocardiography, particularly TEE [11].

Cardiac Computed Tomography (CCT) is an accurate, noninvasive alternative to TEE for the detection of LAA morphology categories and thrombi, distinctly when delayed-imaging acquisition protocols are used but expensive and with high amount of radiation and iodinated contrast [12].

Cardiac Magnetic Resonance Imaging (MRI) might possibly be used instead of TEE or CCT or perhaps three-dimensional echocardiography especially in younger patients with lone $\mathrm{AF}$, at unclear risk for systemic embolism [2, 13].

Cardiac imaging is also of primary importance in the setting of LAA closure devices and electrophysiological studies for AF ablation.

TEE findings of atrial stasis, thrombosis and of aortic atheroma are independently associated with high thromboembolic risk in patients with AF. The increased risk of systemic embolism along with a history of hypertension in AF appears to be mediated primarily through left atrial stasis and thrombi. The presence of complex aortic plaque distinguished patients with $\mathrm{AF}$ at high risk from those at moderate risk of thromboembolism.

\section{Conclusion}

Two-dimensional echocardiography should be recommended in all patients with acute peripheral embolism periprocedural and TEE only in selected cases.

TEE has a diagnostic role, providing detailed information on LA and LAA structure, function and hemodynamic. It guides the intensity of anticoagulation and assesses the indication of LAA closure in special cases. It has prognostic value in patients at low and intermediate risk scores for thromboembolism.

Because we observed a high rate of recurrence for peripheral thromboembolism in our country especially in elderly, it seems necessary to indicate severe anticoagulant therapy although it is difficult because of the risk of both systemic embolism and cerebral hemorrhage that increases with age.

We noticed a higher incidence of acute limb ischemia of embolic rather than atheroatous origin and embolisation is mostly cardiac than arterial (mobile atheroma from aorta). Also, we noticed a very high incidence of acute limb embolism, which is probably due to lack of anticoagulation preoperatively more than due to nontherapeutically anticoagulation $(\mathrm{INR}<2)$. 


\section{References}

1. Maehama $\mathrm{T}$, Okura $\mathrm{H}$, Imai $\mathrm{K}$, et al. Usefulness of CHADS2 score to predict C-reactive protein, left atrial blood stasis, and prognosis in patients with non-rheumatic atrial fibrillation. $A m \mathrm{~J}$ Cardiol 2010; 106(4):535-538.

2. Romero J, Cao JJ, Garcia MJ, Taub CC. Cardiac imaging for assessment of left atrial appendage stasis and thrombosis. Nat Rev Cardiol 2014; 11(8):470-480.

3. Singla V, Singh $Y$, Ravindranath SK, Manjunath CN. 'Bird-beak sign' of left atrial thrombus: a guide to management. BMJ Case Rep, 2013, doi:10.1136/bcr-2013-010459.

4. Sawit ST, Garcia-Alvarez A, Suri B, et al. Usefulness of cardiac computed tomographic delayed contrast enhancement of the left atrial appendage before pulmonary vein ablation. $A m$ J Cardiol 2012; 109(5):677- 684.

5. Lohrmann GM, Peters F, van Riet S, Essop MR. Double Trouble - A Case Report of Mobile Right Atrial Thrombus in the Setting of Acute Pulmonary Thromboembolism. Heart, Lung Circulation 2014; 23:214- 216.

6. Dorenkamp M, Sohns C, Vollmann, D et al. Detection of left atrial thrombus during routine diagnostic work-up prior to pulmonary vein isolation for atrial fibrillation: role of transesophageal echocardiography and multidetector computed tomography. Int $J$ Cardiol 2013; 163(1):26- 33.

7. Tahara A, Tahara N, Honda A, Imaizumi. Mobile left atrial round mass free floating in mitral regurgitation flow mimicking a whale spray. BMJ Case Rep 2014; doi: 10.1136/bcr2013-202725.

8. Black IW. Spontaneous echo contrast: where there's smoke there's fire. Echocardiography 2000; 17(4):373-382.
9. Shirani J, Alaeddini J. Structural remodeling of the left atrial appendage in patients with chronic non-valvular atrial fibrillation: Implications for thrombus formation, systemic embolism, and assessment by transesophageal echocardiography. Cardiovasc Pathol 2000; 9(2):95-101.

10. Zabalgoitia M, Halperin JL, Pearce LA, Blackshear JL, Asinger RW, Hart RG. Transesophageal echocardiographic correlates of clinical risk of thromboembolism in nonvalvular atrial fibrillation. Stroke Prevention in Atrial Fibrillation III Investigators. $J A m$ CollCardiol 1998; 31(7):1622-1626.

11. Cohen A, Chauvel C, Abergel E, Albo C, Benhalima B, Valty J. Value of transesophageal echocardiography in the cardiovascular assessment of an ischemic cerebral accident of suspected embolic origin. Ann Radiol 1994; 37(1-2):29-40.

12. Yamamoto $M$, Seo $Y$, Kawamatsu $N$, et al. Complex left atrial appendage morphology and left atrial appendage thrombus formation in patients with atrial fibrillation. Circ Cardiovasc Imaging 2014; 7(2):337-343.

13. Rathi VK, Reddy ST, Anreddy S, et al. Contrast-enhanced CMR is equally effective as TEE in the evaluation of left atrial appendage thrombus in patients with atrial fibrillation undergoing pulmonary vein isolation procedure. Heart Rhythm 2013; 10(7):1021-1027.

14. Luca C, Ciubotaru A, Leferman C, Anghel R, Deju E, Tinică G. Leriche Syndrome in a postCABG patient. Arch Clin Cases 2015; 2(1):1217. 